\title{
SCIENTIFIC REPORTS

\section{OPEN The DOT1L inhibitor Pinometostat decreases the host-response against infections: Considerations about its use in human therapy}

\begin{abstract}
Laura Marcos-Villar ${ }^{1,2}$ \& Amelia Nieto ${ }^{1,2}$
Patients with acute myeloid leukemia frequently present translocations of $M L L$ gene. Rearrangements of MLL protein (MLL-r) in complexes that contain the histone methyltransferase DOT1L are common, which elicit abnormal methylation of lysine 79 of histone $\mathrm{H} 3$ at $M L L$ target genes. Phase 1 clinical studies with pinometostat (EPZ-5676), an inhibitor of DOT1L activity, demonstrated the therapeutic potential for targeting DOT1L in MLL-r leukemia patients. We previously reported that down-regulation of DOT1L increases influenza and vesicular stomatitis virus replication and decreases the antiviral response. Here we show that DOT1L inhibition also reduces Sendai virus-induced innate response and its overexpression decreases influenza virus multiplication, reinforcing the notion of DOT1L controlling viral replication. Accordingly, genes involved in the host innate response against pathogens ( $R U B I C O N, T R I M 25, B C L 3)$ are deregulated in human lung epithelial cells treated with pinometostat. Concomitantly, deregulation of some of these genes together with that of the MicroRNA let-7B, may account for the beneficial effects of pinometostat treatment in patients with MLL-r involving DOT1L. These results support a possible increased vulnerability to infection in MLL-r leukemia patients undergoing pinometostat treatment. Close follow up of infection should be considered in pinometostat therapy to reduce some severe side effects during the treatment.
\end{abstract}

Acute leukemia is the most frequent cancer and the leading cause of cancer mortality in the pediatric age. Two different types of leukemia have been recognized: acute lymphoblastic leukemia (ALL) and acute myeloid leukemia (AML), the former accounting for around $80 \%$ of all cases ${ }^{1,2}$. Chromosomal translocation is one of the main mechanisms responsible for tumor development and is very common in both types of leukemia ${ }^{3,4}$. One of the most common translocations involves the Mixed Lineage Leukemia $(M L L)$ gene $^{3}$; around $70 \%$ of infant leukemia and $10 \%$ of adult AML contain $M L L$ rearrangements ${ }^{5,6}$.

Translocation of $M L L$ gene generates new $M L L$ fusion genes causing deregulation of genome expression and activation of oncogenes. More than 80 different partner genes have been described to fuse to $M L L$ gene originating different protein complexes. Many of these complexes interact with factors involved in transcriptional regulation such as the histone methylase DOT1L that is frequently found in the complexes ${ }^{6,7}$. DOT1L protein is a methyltransferase that exclusively methylates lysine 79 of histone 3 (H3K79), adding mono- di- or trimethyl groups ${ }^{8}$. Some of the $M L L$-rearranged (MLL-r) leukemia genes are modulated through hypermethylation by DOT1L of a set of genes that elicit leukemogenesis. The pivotal role of DOT1L in MLL-r leukemia and its enzymatic specificity, have favored the search for chemical compounds directed against this molecule for use in leukemia treatment. Among DOT1L inhibitors, pinometostat has acquired a prominent role, since it constitutes a potent and specific inhibitor of DOT1L activity ${ }^{9}$. Treatment with pinometostat results in inhibition of H3K79 methylation and hence $M L L$-fusion genes expression ${ }^{7,9,10}$.

Influenza virus is an RNA virus whose transcription requires short-capped oligonucleotides as primers, which are scavenged from newly synthesized host RNA polymerase II transcripts, ${ }^{11,12}$. This transcription strategy requires, on-going cellular transcription, implies a functional association of virus transcription with host genome expression and thus depends on chromatin dynamic. Looking for epigenetic changes elicited by influenza virus infection, we observed specific changes in the methylation status of lysine 79 of histone 3 (H3K79) in infected

${ }^{1}$ Centro Nacional de Biotecnología (CNB-CSIC), Darwin 3, Cantoblanco, 28049, Madrid, Spain. ${ }^{2}$ CIBER de Enfermedades Respiratorias CIBERES, Madrid, Spain.email: Imarcos@cnb.csic.es; anieto@cnb.csic.es 


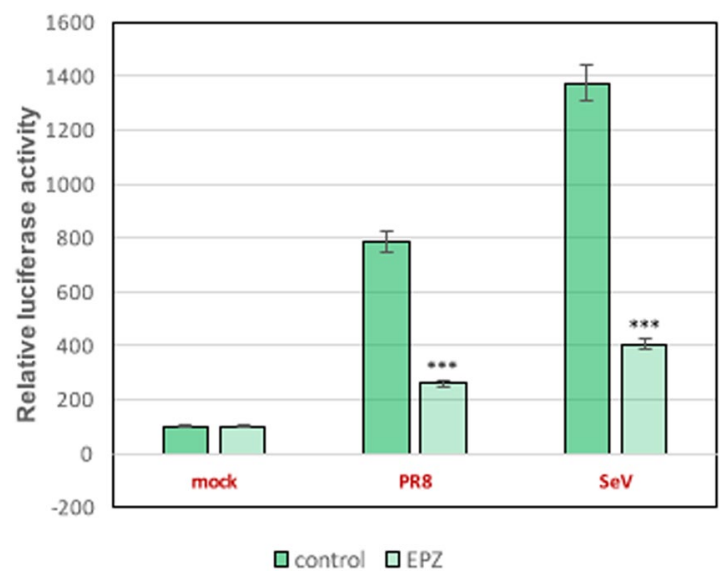

Figure 1. A549 cells untreated (Control) or treated with $1 \mu \mathrm{M}$ pinometostat (EPZ) for $48 \mathrm{~h}$, were transfected with plasmid pIF-lucter and infected with influenza virus or with Sendai virus at $1 \mathrm{pfu} / \mathrm{cell}$. At $16 \mathrm{~h}$ postinfection, luciferase accumulation was monitored by luciferase Assay System (Promega). Three technical replicates of three independent experiments were analyzed. ns $\mathrm{P}>0.05 ; * \mathrm{P}<0.05 ; * * \mathrm{P}<0.01 ; * * * \mathrm{P}<0.001$.

human lung epithelial cells A54913. To characterize the possible contribution of H3K79 methylation to influenza virus control, treatment with pinometostat or/and use of specific lentiviral silencers for DOT1L expression were used. The data indicated that DOT1L down-regulates the antiviral signaling mediated by interferon and thus activates influenza virus replication through decrease of the antiviral response ${ }^{13}$. The effect of pinometostat controlling viral replication may be more general, since it also stimulates viral replication of vesicular stomatitis virus, a potent inducer of interferon ${ }^{13}$. From this study we concluded that methylation of H3K79 by DOT1L has an important role in the control of the interferon signaling and may modulate the infection of different pathogens.

\section{Results}

Effect of pinometostat in the control of interferon signaling. Pinometostat treatment inhibits NF- $\kappa B$ nuclear translocation and reduces the expression of $\beta$-interferon and interferon stimulated genes such as Mx1 or ISG56 in influenza virus infected cells ${ }^{13}$. To further characterize pinometostat effects on IFN signaling, A549 cells untreated or treated with the DOT1L inhibitor for $48 \mathrm{~h}$, were transfected with a plasmid expressing luciferase under the IFN- $\beta$ promoter and infected at 1 plaque-forming units (pfu) per cell with influenza virus (PR8) or with Sendai virus (SeV), an efficient IFN-inducer virus ${ }^{14}$. At $16 \mathrm{~h}$ post-infection, luciferase accumulation was monitored as described in Materials and Methods (Fig. 1). Infection with either influenza or Sendai viruses stimulated luciferase accumulation in comparison with non-infected cells and pinometostat treatment caused a significant decrease of luciferase accumulation in the infected cells, reinforcing the role of DOT1L in controlling interferon signaling.

DOT1L overexpression inhibits influenza virus replication. Down-regulation of DOT1L stimulates influenza and vesicular stomatitis virus replication in cultured cells ${ }^{13}$. The effect of DOT1L overexpression was now examined in A549 transfected cells with a plasmid that expresses human His-DOT1L protein. Pinometostat treated cells during $48 \mathrm{~h}$ (EPZ), untransfected cells (control or Ctl) or cells transfected with the plasmid that expresses His-DOT1L (His-DOT1L) during $48 \mathrm{~h}$, were used to verify DOT1L overexpression and DOT1L enzymatic activity determined by the accumulation of $\mathrm{H} 3 \mathrm{~K} 79 \mathrm{me} 2$, as analyzed by immunoflorescence (Fig. 2A) and Western blot (Fig. 2B). In addition, the accumulation levels of H4K20me 2 were analyzed by immunofluorescence (Fig. 2A) and those of $\mathrm{H} 3$ and H4K16ac by Western blot as controls (Fig. 2B). Cells with up-regulated DOT1L expression were infected with influenza virus at $10^{-3} \mathrm{pfu} / \mathrm{cell}$ (PR8 Dot1L) and viral titters were analyzed by plaque assay at different times post-infection. In parallel, cells were treated with $1 \mu \mathrm{M}$ pinometostat for $48 \mathrm{~h}$ and infected with PR8 under the same conditions (PR8 EPZ). An important and significant decrease in influenza virus replication was observed in A549 cells overexpressing DOT1L and a significant increase was apparent in pinometostat-treated cells (Fig. 2C).

Effect of DOT1L downregulation in the expression of genes that control the host-response against infection in human lung epithelial cells. Next we examined cellular targets methylated at H3K79 by performing RNA-seq analysis of A549 cells with active (control) or inactive DOT1L methylase (treated with $1 \mu \mathrm{M}$ EPZ-5676, from Novagen). At various times post-treatment, the accumulation levels of methylated H3K79 were monitored by Western blot (Fig. 3A), immunofluorescence (Fig. 3B) and a quantitative colorimetric method based on anti-H3K79me2 antibody detection (Epigentek) ${ }^{13}$ (Fig. 3C). An important decrease on H3K79me2 levels was detected using all three different analyses, which indicate the efficiency of the pinometostat treatment. As previously showed ${ }^{13}$, treatment of A549 cells with $1 \mu \mathrm{M}$ pinometostat does not affect cell viability during $48 \mathrm{~h}$, as analyzed by the MTT assay ${ }^{15}$ (Fig. 3D). RNAs isolated from duplicate cultures of untreated or treated cells during $48 \mathrm{~h}$ with pinometostat were used for high-throughput sequencing as described in Materials and Methods. Comparison of RNAs whose expression is modified during the treatment was performed using a 
A
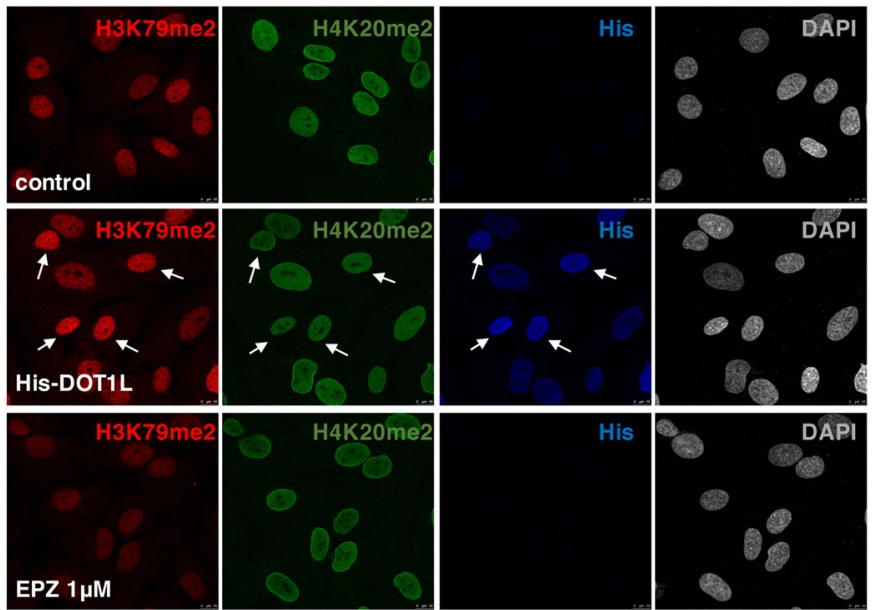

C

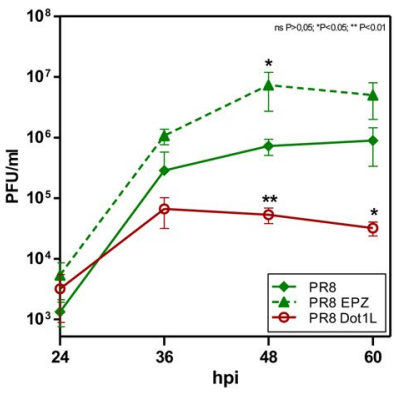

B

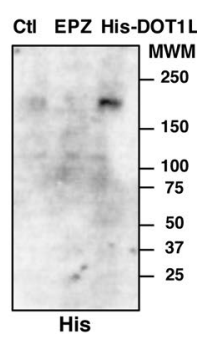

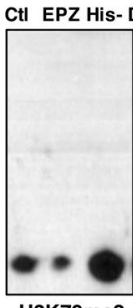

H3K79me2

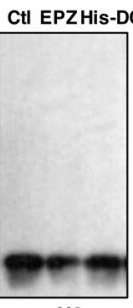

H3

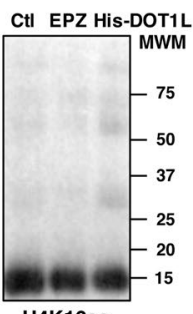

H4K16ac

Figure 2. A549 untransfected cells (control), transfected with plasmid His-Dot1L 48 h (His-DOT1L) or treated with pinometostat $48 \mathrm{~h}$ (EPZ) were used (A) for immunofluorescence analysis against (His), H3K79me2 and H4K20me2. White arrows denote cells with high expression of His-DOT1L. (B) Western blots against HisDOT1L, H3K79me2, H3 and H4K16ac. (C) Untransfected cells (PR8), transfected with His-Dot1L 48 h (PR8 Dot1L) or treated with pinometostat $48 \mathrm{~h}$ (PR8 EPZ), were infected with $10^{-3} \mathrm{pfu} /$ cell with PR8 influenza virus and infective particles analyzed by plaque assay. Three independent experiments were performed. $\mathrm{ns} \mathrm{P}>0.05$; $* \mathrm{P}<0.05 ; * * \mathrm{P}<0.01 ; * * * \mathrm{P}<0.001$.

minimum false-discovery rate (pvalue) of $<0.001$ as statistical significance and $\log 2$ fold change $>2$ as cut-off. Using these parameters, only a few RNAs changed in the presence of the inhibitor (Table 1), suggesting that H3K79 methylation occurs in a limited number of genes in these cells.

In agreement with the role of H3K79 methylation in viral infection, alterations in RUBCN, TRIM25 and BCL3 gene expression in DOT1L down-regulated cells were found. In these conditions, expression of $R U B C N$ and TRIM25 was increased, while a reduction in the expression of $B C L 3$ was found.

- $\quad R U B C N$ gene encodes Rubicon, an important modulator of cellular autophagy; this is a fundamental pathway that eliminates intracellular pathogens through degradation ${ }^{16}$, activates intracellular signaling pathways and stimulates inflammatory mediators ${ }^{17}$. In addition, Rubicon works as negative regulator of type I interferon pathway through interacting with interferon regulatory factor 3 (IRF3) ${ }^{18}$. Down-regulation of Rubicon inhibits influenza and vesicular stomatitis virus replication due to promotion of type I IFN signaling and its overexpression causes the contrary effects ${ }^{18}$.

- Particular attention deserves changes in TRIM25 expression. Thus, RIG-I is a sensor for viruses such as influenza A, Sendai virus, and flaviviruses ${ }^{19-21}$ and its activation is mediated by ubiquitination, which is partly mediated by TRIM $25^{22}$. Accordingly, TRIM 25 has been reported as a host restriction factor for HIV- $1^{23}$ and influenza virus infection ${ }^{22}$.

- $\mathrm{Bcl} 3$ has been identified as an $\operatorname{Ikk} \beta$ protein able to interacts with the p50 and p52 subunits of the NF- $\kappa B^{24,25}$. $\mathrm{Bcl} 3$ is a proto-oncogene that regulates cell proliferation and it is overexpressed in human T-cell leukemia virus infected cells eliciting overgrowth of the $\mathrm{T}$ infected cells ${ }^{26}$. It has been suggested that $\mathrm{Bcl} 3$ modulates NF-kB-dependent gene transcription ${ }^{27,28}$.

To validate the RNA-seq results, the expression of these genes, as well as microRNA let-7B and an additional control gene not regulated by DOT1L ( $M x$ gene) was evaluated by qRT-PCR analysis as described in Materials and Methods. Cultured A549 cells were left untreated or treated with $1 \mu \mathrm{M}$ pinometostat for $48 \mathrm{~h}$, total RNA was isolated and used for qRT-PCR studies. Comparison of the gene expression data obtained by the RNA-seq and qRT-PCR analysis is shown in Fig. 4, where similar values can be observed.

Together, our previous results ${ }^{13}$ and the RNA-seq experiments described here indicate that reduction of H3K79 methylation, alters the expression of several RNAs that encode proteins involved in the signaling pathways that control the host-response against pathogens. 
A

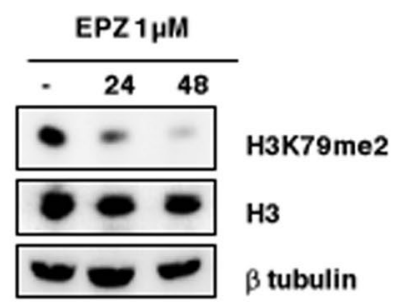

C

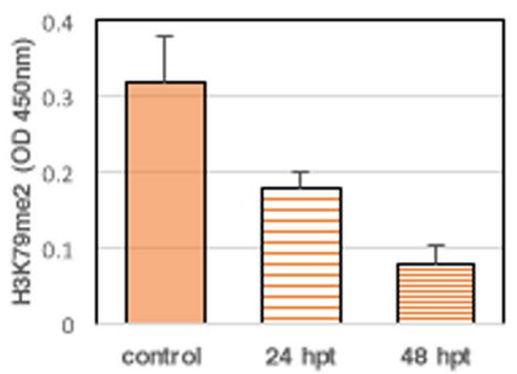

B
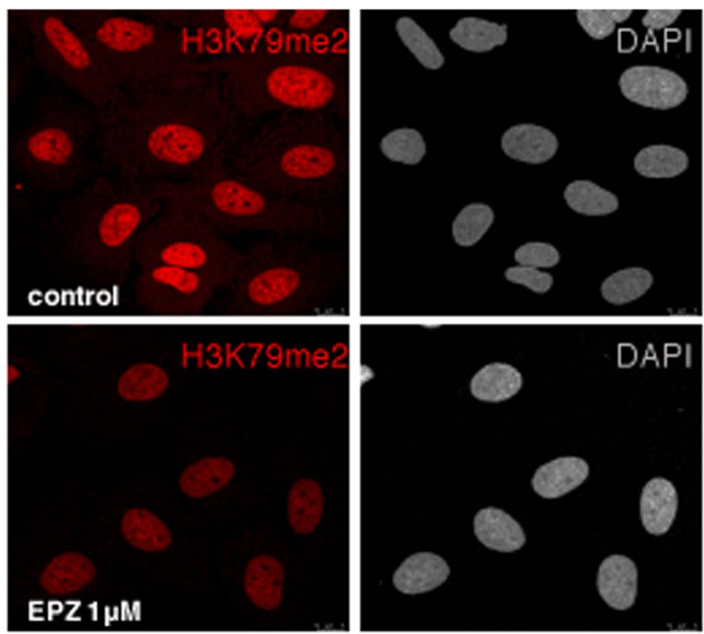

D

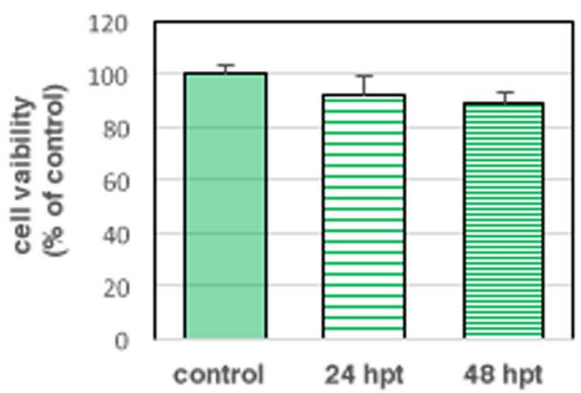

Figure 3. Total extracts of A549 cells untreated (control) or treated with pinometostat $1 \mu \mathrm{M}(\mathrm{EPZ})$ during the indicated times were used for (A) Western blot against the specified proteins, $(\mathbf{B})$ immunofluorescence analysis against H3K79me2 and (C) colorimetric detection of H3K79me2. (D) Viability of A549 cells treated with $1 \mu \mathrm{M}$ DOT1L inhibitor was determined by MTT assay to measure cell metabolic activity.

\begin{tabular}{|l|l|l|}
\hline GENE NAME & Log2FC & DESCRIPTION \\
\hline RUBCN & 5.766742852 & RUN and cysteine rich domain containing beclin 1 interacting protein \\
\hline MIRLET7B & 5.595201384 & microRNA let-7b \\
\hline PGBD2 & 4.909228246 & piggyBac transposable element derived 2 \\
\hline TMEM181 & 4.758097693 & transmembrane protein 181 \\
\hline SBNO1 & 4.547872227 & strawberry notch homolog 1 \\
\hline DGCR8 & 4.43960124 & DGCR8, microprocessor complex subunit \\
\hline C15orf52 & 4.264753684 & chromosome 15 open reading frame 52 \\
\hline REXO1 & 4.215797413 & RNA exonuclease 1 homolog \\
\hline TRIM25 & 3.50263504 & tripartite motif containing 25 \\
\hline DMC1 & 3.188217042 & DNA meiotic recombinase 1 \\
\hline TPR & -2.158545426 & translocated promoter region, nuclear basket protein \\
\hline BCL3 & -2.963329088 & B-cell CLL/lymphoma 3 \\
\hline NFATC2IP & -3.424029921 & nuclear factor of activated T-cells 2 interacting protein \\
\hline PRLH & -7.451413723 & prolactin releasing hormone \\
\hline
\end{tabular}

Table 1. List of proteins modulated by Pinometostat. Duplicate cultures of A549 cells were untreated or treated with pinometostat $1 \mu \mathrm{M}, 48 \mathrm{~h}$ and total RNA was used for RNA-sequencing. The list of proteins encoded by the modulated genes using a qualue $<0.001$ and $\log 2$ fold change $>2$ is shown.

\section{Discussion}

Pinometostat was initially used in preclinical studies in animal models where it showed efficient eradication of leukemia clone from MLL-r leukemia ${ }^{29}$. Pinometostat also showed efficacy as antitumor drug in a xenograft model of MLL-r leukemia ${ }^{10}$ and has been the first member of histone methyltransferase inhibitors used in Phase 1 clinical trials in MLL-r patients, both adult and pediatric. 


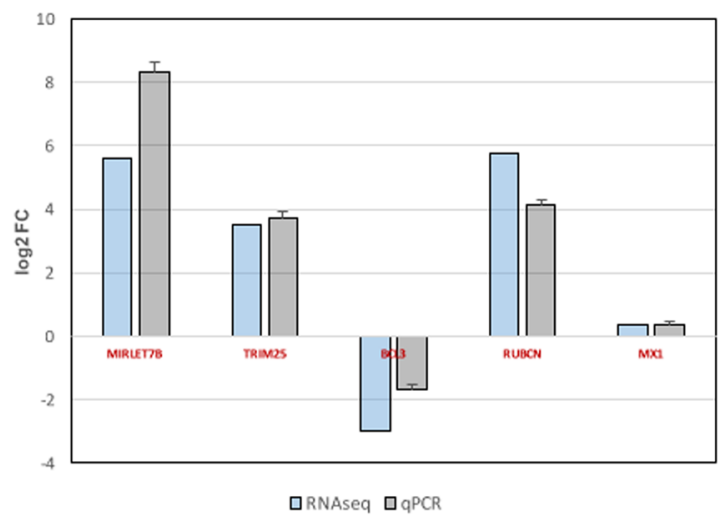

Figure 4. Comparison of RNA-seq and qRT-PCR data of A549 cells treated with pinometostat. For qRT-PCR analysis, A549 cells were plated alone or with pinometostat $(1 \mu \mathrm{M}, 48 \mathrm{~h})$. RNA was extracted and used for qPCR detection of MIRLET7B, TRIM25, BCL3, and RUBICON genes and MX1 as control. Comparison of the qRTPCR and RNA-seq data is shown. The differences between untreated and treated cells were analyzed and are represented as $\log 2$ Fold Change. Three technical replicates of three independent experiments were performed.

As shown in Table 1, pinometostat treatment alters the expression of some genes involved in leukemia control such as MicroRNA let-7B (let-7b) and RUBICON that are up-regulated, or BCL3 that is down-regulated.

Expression of let-7b is remarkably decreased in ALL patients ${ }^{30}$, its potential role in diagnosis and its use as a therapeutic tool for infant ALL treatment have been suggested ${ }^{31,32}$. Down-regulation of DOT1L through pinometostat treatment notably increased MicroRNA let-7b expression in A549 cells (Table 1, Fig. 4). Thus pinometostat administration in patients could contribute to compensate the reduction of this micro RNA observed in ALL patients.

RUBICON expression is significantly decreased in AML cases compared with control group ${ }^{33}$. Accordingly, an involvement of autophagy pathway in the outcome of AML leukemia has been suggested. The increased expression of RUBICON in DOT1L down-regulated cells (Table 1, Fig. 4) could modulate autophagy, restricting the effect on proliferation of myeloid cells in pinometostat treated AML patients.

Treatment with pinometostat decreases BCL3 gene expression (Table 1, Fig. 4). Bcl3 can exist as unphosphorylated form that functions as a conventional inhibitor of $\mathrm{Ikk} \beta$ and promotes NF- $\kappa \mathrm{B}$ activation ${ }^{34}$, and as an isoform phosphorylated by Akt, Erk2 or Ikk1/2 kinases $^{34}$. Phosphorylation of $\mathrm{Bcl} 3$ promotes its nuclear translocation and its conversion into a transcriptional co-regulator of NF- $\kappa \mathrm{B}$, thus functioning as an oncoprotein ${ }^{34}$. Around $40 \%$ of AML patients have constitutively augmented activity of NF- $\kappa B$ and this activity seems to stimulate evasion of apoptosis and cell proliferation ${ }^{35}$. Also, AML CD $34^{+}$cells, express detectable activity of NF- $\kappa B^{36}$, reinforcing the notion that NF- $\kappa B$ signaling has a pivotal role in the development of AML leukemia. Inhibition of DOT1L induces an important decrease on $B C L 3$ expression, a gene with pivotal role controlling NF- $\kappa \mathrm{B}$ activation.

Together, we show here that treatment of human epithelial cells with pinometostat modifies the expression of few cellular genes, being an important number of them involved in the modulation of leukemia altered pathways, such as the microRNA let-7b, RUBICON, or BCL3 genes.

The most common symptoms found in acute myeloid leukemia derive from alterations in blood cells levels, including decrease of neutrophils (neutropenia), which frequently causes fever as a symptom of subsequent infections. Different pathogens including gram negative and positive bacteria, viruses and fungi are responsible for the infection in patients with neutropenia (see for a review ${ }^{37}$ ) and the frequency and causative agent of the infections may depend on the treatment phase ${ }^{38}$.

Pinometostat has been used in Phase 1 clinical trials in MLL-r patients. In pediatric studies, treatment with pinometostat in 18 patients (from 3 months to 18 years of age) showed a transient reduction in peripheral or bone marrow blasts in $40 \%$ of the patients ${ }^{39}$. More than $20 \%$ of patients presented treatment-emergent adverse events (TEAEs) that included febrile neutropenia and respiratory failure among others ${ }^{39}$. In a recent survey of pinometostat treatment a total of 51 adult patients were engaged, 33 of which were AML patients with MLL-r and 4 ALL patients with MLL-r; the total number of patients with MLL-rearrangement represented $72.5 \%$ of the total cohort. Administration of pinometostat was safe, and its efficacy was modest, suggesting a possible therapeutic use of pinometostat when combined with other anti-leukemia agents ${ }^{40}$. The treatment-emergent adverse events of this study were febrile neutropenia (35\%), cough (22\%) and pneumonia (18\%) and serious adverse effects were described in 35 patients (69\%). The most common ones may be related with infection and were distributed as febrile neutropenia (25\%), respiratory failure $(12 \%)$ and pneumonia $(10 \%)^{40}$.

$\mathrm{NF}-\kappa \mathrm{B}$ has a prominent role in the innate immune response against many different pathogens including bacteria, virus and fungi ${ }^{41-44}$. Activation of NF- $\kappa B$ is required to up-regulate apoptosis, Toll-like, RIG-I-like and NOD-like receptor signaling pathways ${ }^{45}$; which are common alterations shared in the host gene expression elicited by different relevant respiratory bacterial and viral pathogens ${ }^{45}$. Pinometostat treatment causes an important reduction on NF- $\kappa B$ nuclear translocation in cells activated with TNF- $\alpha$ or infected with influenza virus ${ }^{13}$. Hence, the serious adverse effects observed in MLL-r patients treated with pinometostat may be partly due to a decreased activation of NF- $\kappa \mathrm{B}$ caused by the drug that would prevent the induction of innate host response and increase pathogen amplification. 
Future directions. Leukemia patients are particularly prone to pathogen infections. As shown above, pinometostat decreases signaling pathways that control the innate immune response against infections. Treatment with pinometostat is associated with a number of side effects that may derive from infections. Preventive administration of antibiotics or/and early survey of infections mediated by bacteria, virus or fungi should be considered concomitantly with pinometostat therapy to diminish severe side effects elicited by the treatment.

\section{Materials and Methods}

Western blotting. Western blotting was carried out as described previously ${ }^{46}$. The following antibodies were used: for H3K79me2 a rabbit polyclonal antibody (1:1000) (D15E8), for H3 a rabbit polyclonal antibody (1:1000) $(\mathrm{D} 1 \mathrm{H} 2)$, and for H4K16ac a rabbit polyclonal antibody (1:1000) (E2B8W) from Cell Signaling. For DOT1L detection, a rabbit polyclonal antibody anti-His (1:600) and for $\beta$-tubulin a mouse monoclonal antibody (1:1000), both from Sigma.

Confocal immunofluorescence microscopy. A549 cells were used for immunofluorescence assays as described $^{13}$ using a polyclonal antibody against H3K79me2 (1:250) from Cell Signaling, a polyclonal antibody against H4K20me2 (1:200) (NB100-66606) from Nobus and a monoclonal antibody anti-His (1:200) (631212) from Clontech for His-DOT1L detection with a Leica TCS SP5 laser scanning system.

Transfection-infection experiments. A549 cells were left untransfected or transfected with a plasmid expressing His-DOT1L (Addgene) for $48 \mathrm{~h}$. The cells were then infected with the A/Puerto Rico/8/34 (PR8) strain of influenza virus at $10^{-3} \mathrm{pfu} / \mathrm{cell}$ and virus multiplication was analyzed by plaque assay. Cells treated with $1 \mu \mathrm{M}$ pinometostat (EPZ) for $48 \mathrm{~h}$, and infected with PR8 at the conditions indicated above, were carried out in parallel. Three independent experiments were performed.

Human A549 cells were untreated (mock) or treated with $1 \mu \mathrm{M}$ pinometostat (EPZ) for $48 \mathrm{~h}(\mathrm{EPZ})$, and then transfected with a luciferase reporter plasmid containing an IFN- $\beta$ promoter (pIF-lucter), using VIROMER RED ONE. At $16 \mathrm{~h}$ post-transfection, cells were either mock infected or infected with PR8 or with Sendai virus (SeV) at $1 \mathrm{pfu} / \mathrm{cell}$. At $16 \mathrm{~h}$ post-infection, luciferase accumulation was monitored using the luciferase Assay System (Promega). Three technical replicates of three independent experiments were analyzed.

In all transfection experiments, an amount of DMSO equivalent to the amount of this solvent in the pinometostat treated cells, was added to the untreated cells. A plasmid expressing renilla luciferase was added to the mixture in all transfection experiments to normalize transfection efficiency. Significance was determined by two way ANOVA analysis; $(* P<0.5 ; * * P<0.01 ; * * * P<0.001)$.

RNA seq. Total RNAs were extracted using Trizol reagent (Invitrogen) according to the manufacturer's instructions from control or pinometostat treated cells. Duplicate cultures of untreated or treated cells with $1 \mu \mathrm{M}$ pinometostat during $48 \mathrm{~h}$ were used for high-throughput sequencing with TruSeq v3 chemistry and 50 bp single reads on an Illumina HiSeq. 2000. For RNA-seq analysis, sequenced reads were aligned to the Homo sapiens genome (version GRCh38.p8 from NCBI) using TopHat 2.1.1 ${ }^{47}$ linked to Bowtie 2.2.8 ${ }^{48}$ with default sensitive settings. From sequenced reads, transcripts were assembled using Cufflinks 2.2.1 ${ }^{49}$ and differential expression analyses were performed with Cuffdiff $2.2 .1^{50}$. Additional information for each gene was obtained from the NCBI database and included in the dataset. Genes with expression levels under a threshold in both control and treatment conditions were discarded. The median of the distribution of the non-zero values was taken as the threshold. The generated data were uploaded to the FIESTA viewer FIESTA@BioinfoGP for visualization and further filtering

qRT-PCR analysis. For RNA extraction, cell pellets of untreated or EPZ-treated cells with $1 \mu \mathrm{M}$ pinometostat during $48 \mathrm{~h}$, were resuspended in $1 \mathrm{ml}$ of TRIZOL reagent (Invitrogen) and the RNA was purified as recommended by the manufacturer. Reverse transcription was performed using the High-Capacity cDNA RT kit from Applied Biosystems. PCR was performed using SYBR green PCR master mix (Applied Biosystems) and specific primers.

\section{Data availability}

The datasets generated during the current study are deposited in Sequence Read Archive (SRA) of NCBI, with the following codes: BioProject; PRJNA562125, Study: SRP219262. https://dataview.ncbi.nlm.nih.gov/object/ PRJNA562125? reviewer $=$ bj3spmb63srg6bp6ab4j3ka78g.

Received: 4 July 2019; Accepted: 23 October 2019;

Published online: 14 November 2019

\section{References}

1. Hilden, J. M. et al. MLL gene rearrangement, cytogenetic 11q23 abnormalities, and expression of the NG2 molecule in infant acute myeloid leukemia. Blood 89, 3801-3805 (1997).

2. Chowdhury, T. \& Brady, H. J. Insights from clinical studies into the role of the MLL gene in infant and childhood leukemia. Blood Cells Mol Dis 40, 192-199, https://doi.org/10.1016/j.bcmd.2007.07.005 (2008).

3. Muntean, A. G. \& Hess, J. L. The pathogenesis of mixed-lineage leukemia. Annu Rev Pathol 7, 283-301, https://doi.org/10.1146/ annurev-pathol-011811-132434 (2012).

4. Sanjuan-Pla, A. et al. Revisiting the biology of infant $\mathrm{t}(4 ; 11) /$ MLL-AF4+ B-cell acute lymphoblastic leukemia. Blood 126, 2676-2685, https://doi.org/10.1182/blood-2015-09-667378 (2015)

5. Armstrong, S. A., Golub, T. R. \& Korsmeyer, S. J. MLL-rearranged leukemias: insights from gene expression profiling. Semin Hematol 40, 268-273 (2003).

6. Winters, A. C. \& Bernt, K. M. MLL-Rearranged Leukemias-An Update on Science and Clinical Approaches. Front Pediatr 5, 4, https://doi.org/10.3389/fped.2017.00004 (2017). 
7. Wong, M., Polly, P. \& Liu, T. The histone methyltransferase DOT1L: regulatory functions and a cancer therapy target. Am J Cancer Res 5, 2823-2837 (2015).

8. Nguyen, A. T. \& Zhang, Y. The diverse functions of Dot1 and H3K79 methylation. Genes Dev 25, 1345-1358, https://doi.org/10.1101/ gad.2057811 (2011).

9. Daigle, S. R. et al. Potent inhibition of DOT1L as treatment of MLL-fusion leukemia. Blood 122, 1017-1025, https://doi.org/10.1182/ blood-2013-04-497644 (2013).

10. Waters, N. J. Preclinical Pharmacokinetics and Pharmacodynamics of Pinometostat (EPZ-5676), a First-in-Class, Small Molecule S-Adenosyl Methionine Competitive Inhibitor of DOT1L. Eur J Drug Metab Pharmacokinet 42, 891-901, https://doi.org/10.1007/ s13318-017-0404-3 (2017).

11. Resa-Infante, P., Jorba, N., Coloma, R. \& Ortin, J. The influenza virus RNA synthesis machine: advances in its structure and function. RNA Biol 8, 207-215 (2011).

12. Rodriguez-Frandsen, A., Alfonso, R. \& Nieto, A. Influenza virus polymerase: Functions on host range, inhibition of cellular response to infection and pathogenicity. Virus research, https://doi.org/10.1016/j.virusres.2015.03.017 (2015).

13. Marcos-Villar, L. et al. Epigenetic control of influenza virus: role of H3K79 methylation in interferon-induced antiviral response. Sci Rep 8, 1230, https://doi.org/10.1038/s41598-018-19370-6 (2018).

14. Bedsaul, J. R., Zaritsky, L. A., Zoon, K. C. \& Type, I. Interferon-Mediated Induction of Antiviral Genes and Proteins Fails to Protect Cells from the Cytopathic Effects of Sendai Virus Infection. J Interferon Cytokine Res 36, 652-665, https://doi.org/10.1089/ jir.2016.0051 (2016)

15. Levitz, S. M. \& Diamond, R. D. A rapid colorimetric assay of fungal viability with the tetrazolium salt MTT. J Infect Dis 152, 938-945 (1985).

16. Sun, Q. et al. The RUN domain of rubicon is important for hVps34 binding, lipid kinase inhibition, and autophagy suppression. J Biol Chem 286, 185-191, https://doi.org/10.1074/jbc.M110.126425 (2011).

17. Deretic, V., Saitoh, T. \& Akira, S. Autophagy in infection, inflammation and immunity. Nat Rev Immunol 13, 722-737, https://doi. org/10.1038/nri3532 (2013).

18. Kim, J. H. et al. Rubicon Modulates Antiviral Type I Interferon (IFN) Signaling by Targeting IFN Regulatory Factor 3 Dimerization. J Virol 91, https://doi.org/10.1128/JVI.00248-17 (2017)

19. Weber-Gerlach, M. \& Weber, F. Standing on three legs: antiviral activities of RIG-I against influenza viruses. Curr Opin Immunol 42, 71-75, https://doi.org/10.1016/j.coi.2016.05.016 (2016).

20. Sanchez-Aparicio, M. T. et al. Loss of Sendai virus C protein leads to accumulation of RIG-I immunostimulatory defective interfering RNA. J Gen Virol 98, 1282-1293, https://doi.org/10.1099/jgv.0.000815 (2017).

21. Guo, H. Y., Zhang, X. C. \& Jia, R. Y. Toll-Like Receptors and RIG-I-Like Receptors Play Important Roles in Resisting Flavivirus. J Immunol Res 2018, 6106582, https://doi.org/10.1155/2018/6106582 (2018).

22. Gack, M. U. et al. Influenza A virus NS1 targets the ubiquitin ligase TRIM25 to evade recognition by the host viral RNA sensor RIG-I. Cell Host Microbe 5, 439-449, https://doi.org/10.1016/j.chom.2009.04.006 (2009).

23. Stremlau, M. et al. The cytoplasmic body component TRIM5alpha restricts HIV-1 infection in Old World monkeys. Nature 427, 848-853, https://doi.org/10.1038/nature02343 (2004).

24. Hatada, E. N. et al. The ankyrin repeat domains of the NF-kappa B precursor p105 and the protooncogene bcl-3 act as specific inhibitors of NF-kappa B DNA binding. Proc Natl Acad Sci USA 89, 2489-2493, https://doi.org/10.1073/pnas.89.6.2489 (1992).

25. Wulczyn, F. G., Naumann, M. \& Scheidereit, C. Candidate proto-oncogene bcl-3 encodes a subunit-specific inhibitor of transcription factor NF-kappa B. Nature 358, 597-599, https://doi.org/10.1038/358597a0 (1992).

26. Saito, K., Saito, M., Taniura, N., Okuwa, T. \& Ohara, Y. Activation of the PI3K-Akt pathway by human T cell leukemia virus type 1 (HTLV-1) oncoprotein Tax increases Bcl3 expression, which is associated with enhanced growth of HTLV-1-infected T cells. Virology 403, 173-180, https://doi.org/10.1016/j.virol.2010.04.018 (2010).

27. Inoue, J., Takahara, T., Akizawa, T. \& Hino, O. Bcl-3, a member of the I kappa B proteins, has distinct specificity towards the Rel family of proteins. Oncogene 8, 2067-2073 (1993).

28. Nolan, G. P. et al. The bcl-3 proto-oncogene encodes a nuclear I kappa B-like molecule that preferentially interacts with NF-kappa B p50 and p52 in a phosphorylation-dependent manner. Mol Cell Biol 13, 3557-3566, https://doi.org/10.1128/mcb.13.6.3557 (1993).

29. Stein, E. \& Tallman, M. Mixed lineage rearranged leukaemia: pathogenesis and targeting DOT1L. Curr Opin Hematol 22, 92-96 (2015).

30. Mi, S. et al. MicroRNA expression signatures accurately discriminate acute lymphoblastic leukemia from acute myeloid leukemia. Proc Natl Acad Sci USA 104, 19971-19976, https://doi.org/10.1073/pnas.0709313104 (2007).

31. Nishi, M. et al. Suppression of the let-7b microRNA pathway by DNA hypermethylation in infant acute lymphoblastic leukemia with MLL gene rearrangements. Leukemia 27, 389-397, https://doi.org/10.1038/leu.2012.242 (2013).

32. Zhu, Y. D. et al. Distinctive microRNA signature is associated with the diagnosis and prognosis of acute leukemia. Med Oncol 29, 2323-2331, https://doi.org/10.1007/s12032-011-0140-5 (2012).

33. Liang, P. Q. et al. Expression of autophagy genes in acute myeloid leukemia: associations with clinical characteristics and prognosis. Neoplasma 65, 807-814, https://doi.org/10.4149/neo_2018_171028N691 (2018).

34. Wang, V. Y. et al. Bcl3 Phosphorylation by Akt, Erk2, and IKK Is Required for Its Transcriptional Activity. Mol Cell 67, $484-497$ e485, https://doi.org/10.1016/j.molcel.2017.06.011 (2017).

35. Zhou, J., Ching, Y. Q. \& Chng, W. J. Aberrant nuclear factor-kappa B activity in acute myeloid leukemia: from molecular pathogenesis to therapeutic target. Oncotarget 6, 5490-5500, https://doi.org/10.18632/oncotarget.3545 (2015).

36. Guzman, M. L. et al. Nuclear factor-kappaB is constitutively activated in primitive human acute myelogenous leukemia cells. Blood 98, 2301-2307 (2001)

37. Chandra, R., Hakki, M. \& Spurgeon, S. In Sepsis (ed. Luciano Azevedo) https://doi.org/10.5772/50193 (2012).

38. Caniza, M. A. et al. Infectious complications in children with acute lymphoblastic leukemia treated in low-middle-income countries. Expert Rev Hematol 8, 627-645, https://doi.org/10.1586/17474086.2015.1071186 (2015).

39. Shukla, N. et al. Final Report of Phase 1 Study of the DOT1L Inhibitor, Pinometostat (EPZ-5676), in Children with Relapsed or Refractory MLL-r Acute Leukemia. Blood 128, 2780 (2016).

40. Stein, E. M. et al. The DOT1L inhibitor pinometostat reduces H3K79 methylation and has modest clinical activity in adult acute leukemia. Blood 131, 2661-2669, https://doi.org/10.1182/blood-2017-12-818948 (2018).

41. Jenner, R. G. \& Young, R. A. Insights into host responses against pathogens from transcriptional profiling. Nat Rev Microbiol 3, 281-294, https://doi.org/10.1038/nrmicro1126 (2005).

42. Beug, S. T., Cheung, H. H., LaCasse, E. C. \& Korneluk, R. G. Modulation of immune signalling by inhibitors of apoptosis. Trends Immunol 33, 535-545, https://doi.org/10.1016/j.it.2012.06.004 (2012).

43. Newton, K. \& Dixit, V. M. Signaling in innate immunity and inflammation. Cold Spring Harb Perspect Biol, 4, https://doi. org/10.1101/cshperspect.a006049 (2012).

44. Underhill, D. M. \& Pearlman, E. Immune Interactions with Pathogenic and Commensal Fungi: A Two-Way Street. Immunity 43, 845-858, https://doi.org/10.1016/j.immuni.2015.10.023 (2015).

45. Martinez, I. et al. Apoptosis, Toll-like, RIG-I-like and NOD-like Receptors Are Pathways Jointly Induced by Diverse Respiratory Bacterial and Viral Pathogens. Front Microbiol 8, 276, https://doi.org/10.3389/fmicb.2017.00276 (2017). 
46. Rodriguez, A., Pérez-Gonzalez, A. \& Nieto, A. Influenza virus infection causes specific degradation of the largest subunit of cellular RNA polymerase II. J Virol 81, 5315-5324 (2007).

47. Kim, D. et al. TopHat2: accurate alignment of transcriptomes in the presence of insertions, deletions and gene fusions. Genome Biol 14, R36, https://doi.org/10.1186/gb-2013-14-4-r36 (2013).

48. Langmead, B. \& Salzberg, S. L. Fast gapped-read alignment with Bowtie 2. Nat Methods 9, 357-359, https://doi.org/10.1038/ nmeth.1923 (2012).

49. Trapnell, C. et al. Transcript assembly and quantification by RNA-Seq reveals unannotated transcripts and isoform switching during cell differentiation. Nat Biotechnol 28, 511-515, https://doi.org/10.1038/nbt.1621 (2010)

50. Trapnell, C. et al. Differential analysis of gene regulation at transcript resolution with RNA-seq. Nat Biotechnol 31, 46-53, https://doi. org/10.1038/nbt.2450 (2013).

\section{Acknowledgements}

We gratefully acknowledge the assistance of J. Diaz-Colunga in the bioinformatics analyses and the scientific advice of M. Yabe is appreciated. The editorial assistance of J. Ortín is also appreciated. This work was supported by the Spanish Ministry of Economy and Competitivity (BFU2017-83392-R; AEI/FEDER, UE) and Ciber de Enfermedades Respiratorias (CIBERES).

\section{Author contributions}

L.M.-V. was involved in Conceptualization, Data curation, Formal analysis, Methodology, and Validation, A.N. was involved in Conceptualization, Formal analysis, Funding acquisition, Investigation, Project administration, Resources, Supervision, Validation, and Writing.

\section{Competing interests}

The authors declare no competing interests.

\section{Additional information}

Correspondence and requests for materials should be addressed to L.M.-V. or A.N.

Reprints and permissions information is available at www.nature.com/reprints.

Publisher's note Springer Nature remains neutral with regard to jurisdictional claims in published maps and institutional affiliations.

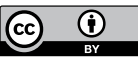

Open Access This article is licensed under a Creative Commons Attribution 4.0 International License, which permits use, sharing, adaptation, distribution and reproduction in any medium or format, as long as you give appropriate credit to the original author(s) and the source, provide a link to the Creative Commons license, and indicate if changes were made. The images or other third party material in this article are included in the article's Creative Commons license, unless indicated otherwise in a credit line to the material. If material is not included in the article's Creative Commons license and your intended use is not permitted by statutory regulation or exceeds the permitted use, you will need to obtain permission directly from the copyright holder. To view a copy of this license, visit http://creativecommons.org/licenses/by/4.0/.

(c) The Author(s) 2019 